\title{
Correction to: Generation of Liver Disease-Specific Induced Pluripotent Stem Cells along with Efficient Differentiation to Functional Hepatocyte-like Cells
}

\author{
Arefeh Ghodsizadeh ${ }^{1,2} \cdot$ Adeleh Taei $^{1} \cdot$ Mehdi Totonchi $^{3} \cdot$ Ali Seifinejad $^{1} \cdot$ Hamid Gourabi $^{3} \cdot$ Behshad Pournasr $^{1}$. \\ Nasser Aghdami ${ }^{1,4} \cdot$ Reza Malekzadeh $^{5} \cdot$ Navid Almadani $^{3} \cdot$ Ghasem Hosseini Salekdeh $^{6} \cdot$ Hossein Baharvand $^{1,7}$
}

Published online: 6 April 2021

(C) Springer Science+Business Media, LLC, part of Springer Nature 2021

\section{Correction to: Stem Cell Rev Rep. 2010 Dec;6(4):622-32} https://doi.org/10.1007/s12015-010-9189-3

The authors regret to acknowledge that a non-intentional human error related to data handling/labelling during the preparation of the hepatic differentiation images in Fig. 4B resulted in an incorrect figure panel placement such that the CYP1A1 images of two iPS cell lines were derived from the same image. We therefore removed the "CYP1A1" column from Fig. 4B of our manuscript. A replacement to Fig. $4 \mathrm{~B}$ is included below.

Although we are very disappointed by this error, we would like to emphasize that the main point of this figure (i.e., that these cell lines have potential for hepatic differentiation) was also separately confirmed with expression of AFP, ALB, HNF $4 \alpha$, and CYP3a4 at mRNA level (Fig. 3a and 4a) and ALB and CK18 at protein level (Fig. $4 b$ and c). Their functionality was also confirmed by glycogen and lipid storage activity, secretion of albumin, alpha-fetoprotein, and urea, CYP450 metabolic activity, as well as LDL and indocyanin green uptake (Fig. 5).

The authors would like to apologise for any inconvenience caused.

The online version of the original article can be found at https://doi.org/ 10.1007/s12015-010-9189-3

Hossein Baharvand

Baharvand@RoyanInstitute.org

1 Department of Stem Cells and Developmental Biology, Royan Institute for Stem Cell Biology and Technology, ACECR, P.O. Box 19395-4644, Tehran, Iran

2 Department of Biotechnology, College of Science, University of Tehran, Tehran, Iran

3 Department of Genetics, Royan Institute for Reproductive Biomedicine, ACECR, Tehran, Iran

4 Department of Regenerative Biomedicine, Royan Institute for Stem Cell Biology and Technology, ACECR, Tehran, Iran

5 Digestive Disease Research Center, Shariati Hospital, Tehran University of Medical Sciences, Tehran, Iran

6 Department of Molecular Systems Biology, Royan Institute for Stem Cell Biology and Technology, ACECR, Tehran, Iran

7 Department of Developmental Biology, University of Science and Culture, ACECR, Tehran, Iran 
a

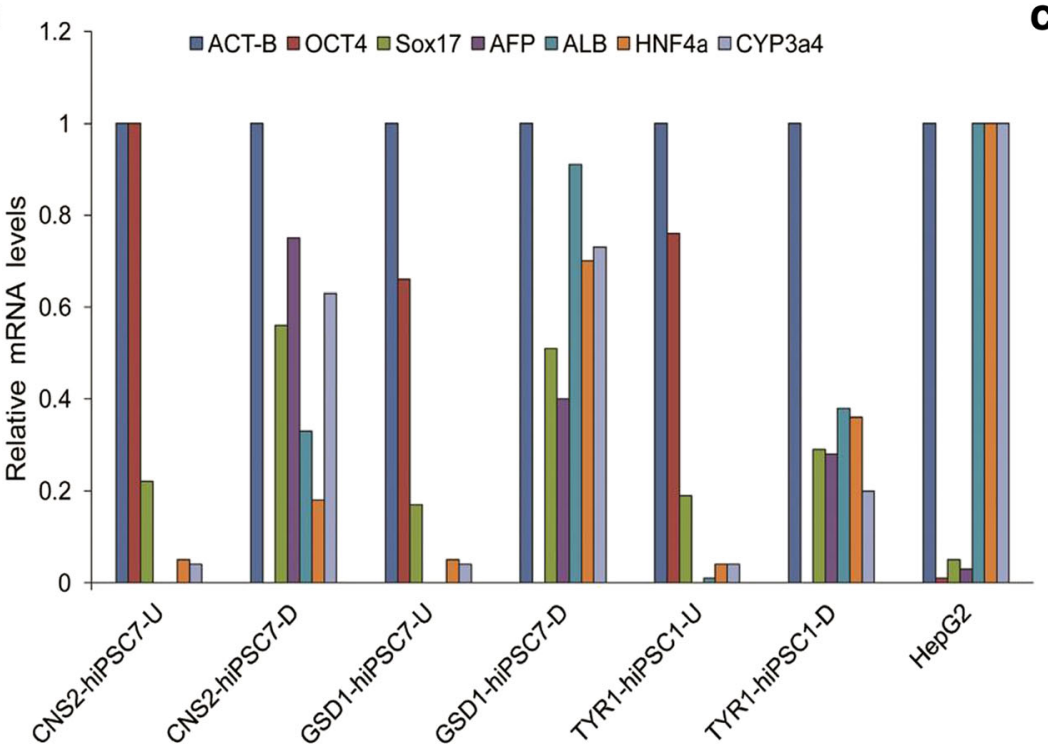

C
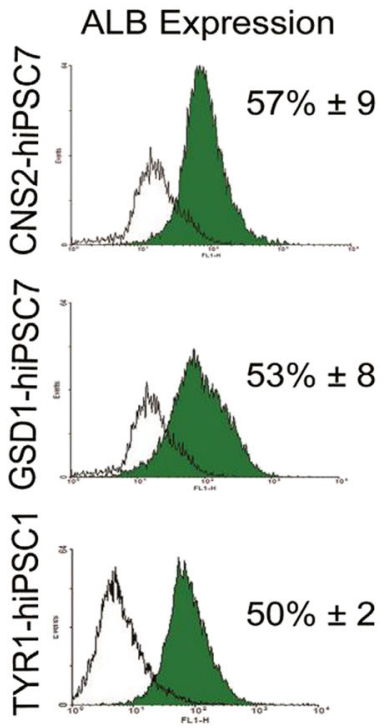

b
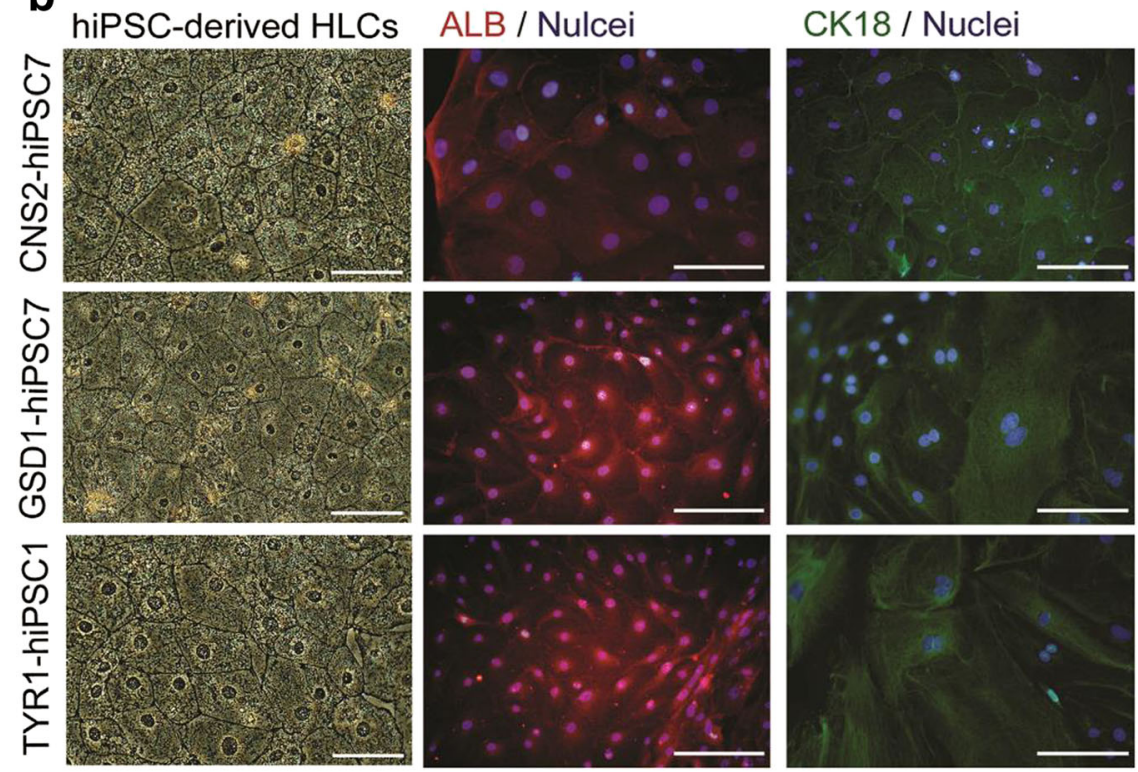

Publisher's Note Springer Nature remains neutral with regard to jurisdictional claims in published maps and institutional affiliations. 